\title{
Improvement in circulating endothelial progenitor cells pool after cardiac resynchronization therapy: increasing the list of benefits
}

\section{Gonçalo Cristóvão}

Centro Hospitalar e Universitario de Coimbra EPE

James Milner

Centro Hospitalar e Universitario de Coimbra EPE

Pedro Sousa

Centro Hospitalar e Universitario de Coimbra EPE

Miguel Ventura

Centro Hospitalar e Universitario de Coimbra EPE

João Cristóvão

Centro Hospitalar e Universitario de Coimbra EPE

Luís Elvas

Centro Hospitalar e Universitario de Coimbra EPE

Artur Paiva

Centro Hospitalar e Universitario de Coimbra EPE

Lino Gonçalves

Universidade de Coimbra Faculdade de Medicina

Carlos Fontes Ribeiro

Universidade de Coimbra Faculdade de Medicina

Natália António ( $\square$ natalia.antonio@gmail.com )

Universidade de Coimbra Faculdade de Medicina https://orcid.org/0000-0003-3103-4353

\section{Research}

Keywords: Endothelial Progenitor Cells, Cardiac Resynchronization Therapy, Heart Failure, Prognosis

Posted Date: March 10th, 2020

DOI: https://doi.org/10.21203/rs.3.rs-16519/v1

License: (c) (1) This work is licensed under a Creative Commons Attribution 4.0 International License.

Read Full License 
Version of Record: A version of this preprint was published at Stem Cell Research \& Therapy on May 24th, 2020. See the published version at https://doi.org/10.1186/s13287-020-01713-8. 


\section{Abstract}

Background Recent studies suggest that circulating endothelial progenitor cells (EPCs) may influence the response to cardiac resynchronization therapy (CRT). The aim of this study was to evaluate the effect of CRT on EPCs levels and to assess the impact of EPCs on long-term clinical outcomes.

Population and methods Prospective study of 50 patients submitted to CRT. Two populations of circulating EPCs were quantified previously to CRT implantation: CD34 + KDR + and CD133 + KDR + cells. EPCs levels were reassessed 6 months after CRT. Endpoints during the long-term follow-up were all-cause mortality, heart transplantation and hospitalization for heart failure (HF) management.

Results The proportion of non-responders to CRT was $42 \%$ and tended to be higher in patients with an ischemic vs non-ischemic etiology ( $64 \%$ vs $35 \%, \mathrm{p}=0.098)$. Patients with ischemic cardiomyopathy (ICM) showed significantly lower CD34+KDR+ EPCs levels when compared to non-ischemic dilated cardiomyopathy patients (DCM) $(0.0010 \pm 0.0007$ vs $0.0030 \pm 0.0024$ cells/ 100 leukocytes, $p=0.032)$. There were no significant differences in baseline EPCs levels between survivors and non-survivors nor between patients who were rehospitalized for HF management during follow-up or not. At 6-month followup, circulating EPCs levels were significantly higher than baseline levels $(0.0024 \pm 0.0023$ vs $0.0047 \pm$ $0.0041 \mathrm{CD} 34+\mathrm{KDR}+$ cells $/ 100$ leukocytes, $p=0.010$ and $0.0007 \pm 0.0004$ vs 0.0016 vs 0.0013 CD133 + $/ K D R+$ cells/100 leukocytes, $p=0.007)$.

Conclusions Patients with ICM showed significantly lower levels of circulating EPCs when compared to their counterparts. CRT seems to improve the pool of endogenously circulating EPCs and reduced baseline EPCs levels seem not influence long-term outcomes after CRT.

\section{Introduction}

Advanced heart failure (HF) is associated with endothelial dysfunction which negatively impacts cardiac function, heart failure progression and survival. ${ }^{1,2}$ Circulating endothelial progenitor cells (EPCs) contribute to endothelial homeostasis and may serve as a circulating reservoir for endothelial repair in various pathological conditions. ${ }^{3}$ Accumulating evidence shows that reduced circulating EPCs levels accurately reflect endothelial dysfunction. ${ }^{4}$

In patients with coronary artery disease, reduced EPCs levels have been identified as an independent predictor of future cardiovascular events. ${ }^{5,6}$ However, in advanced HF, the association between circulating EPCs and the subsequent long-term clinical outcome remains undefined.

Cardiac resynchronization therapy (CRT) is a well-recognized and important treatment for patients with advanced HF. ${ }^{7}$ However, some patients do not respond positively to CRT. Previous studies suggest that endothelial dysfunction may hamper response to CRT. ${ }^{8}$ Moreover, previous work by our group suggest that circulating EPC levels may influence CRT response. ${ }^{9}$ Nevertheless, no previous studies have 
specifically focused on the relation of circulating EPCs to subsequent long-term outcomes of advanced HF patients submitted to CRT nor about the effect of CRT on circulating EPCs levels.

The primary objective of this study was to analyse the impact of CRT on circulating EPCs pool. As a secondary objective we intended to assess the potential value of circulating EPCs as a predictor of longterm clinical outcomes after CRT.

\section{Population And Methods Study Population}

This is a prospective study of 50 patients with advanced HF undergoing cardiac resynchronization therapy (CRT) between 11/2009 and 10/2011 in a single centre. Demographic, clinical parameters (including New York Heart Association [NYHA] classification) and echocardiographic parameters of each patient were assessed before and 6 months after CRT. All patients were under stable, optimized medical therapy for $\mathrm{CHF}$ at the time of inclusion.

Inclusion criteria were a left ventricular ejection fraction (LVEF) $\leq 35 \%$, QRS $\geq 120$ ms with a left bundle branch block morphology and presence of sinus rhythm.

Exclusion criteria were: congenital heart disease, severe valvular disease, acute coronary syndrome, or percutaneous coronary intervention within the preceding 3 months, myocardial revascularization surgery or implantation of a previous cardiac pacing device, severe peripheral arterial occlusive disease, anaemia (haemoglobin $<8.5 \mathrm{~g} / \mathrm{dL}$ ), renal insufficiency (creatinine $>2.0 \mathrm{mg} / \mathrm{dL}$ ) or severe, noncontrolled, arterial hypertension (systolic blood pressure $>180 \mathrm{mmHg}$ or diastolic $>110 \mathrm{mmHg}$ ), recent major bleeding requiring blood transfusion ( 6 months), concomitant inflammatory or infectious disease, autoimmune or neoplastic disease, trauma or surgery in the last month, cardiogenic shock, pregnancy, patients taking regular non-steroidal anti-inflammatory drugs or patients taking vasoactive amines or anticoagulants, comorbidities associated with a life expectancy of less than 1 year, and excessive alcohol consumption or illicit drugs abuse.

\section{Echocardiographic Evaluation}

Standard echocardiography was performed using Vivid 7 echocardiographs (GE Healthcare, Oslo, Norway) and a 1.7-3.4 MHz tissue harmonic transducer; appropriate software was used (EchoPAC, GE Healthcare). Left ventricular end-systolic volume (LVESV), left ventricular end-diastolic volume (LVEDV) and LVEF were calculated by the biplane Simpson's equation in apical four-chamber and two-chamber views.

\section{Long-term follow-up}

Data on mortality, heart transplantation and hospitalization due to worsening heart failure were collected from reviewing hospital records at the closure of the study (April 2018). The echocardiogram performed 
6-months after the implantation was used to assess response to CRT. Patients who demonstrated at least a $15 \%$ reduction in LVESV at the 6-month follow up were defined as responders to CRT.

\section{Quantification of circulating EPCs by flow cytometry}

Blood samples were collected to evaluate the analytical parameters (including brain natriuretic peptide [BNP]), just before the device implantation. In addition, venous blood samples, stored in ethylenediaminetetraacetic acid (EDTA) tubes, were also collected for quantification of circulating EPCs levels, and processed within 1 to 2 hours after collection. Hence, $150 \mu$ of whole blood were incubated with 3 antihuman monoclonal antibody (mAB): $10 \mu$ of APC-conjugated CD133 (Miltenyi Biotec Inc., Auburn, CA, USA), $10 \mu$ l of phycoerythrin (PE) conjugated KDR mAB (type 2 vascular endothelial growth factor receptor - VEGF-R2) (Sigma-Aldrich Co., St. Louis, MO, USA), $10 \mu$ of fluorescein isothiocyanate (FITC) conjugated CD34 mAB (Becton Dickinson and Co.) for 30 min at $4{ }^{\circ} \mathrm{C}$, in the dark. For erythrocyte lysis FACS Lysing Solution (BD Biosciences) diluted in a ratio of 1:10 (vol/vol) with distilled water was used. Subsequently a wash with phosphate buffered saline (PBS) was performed. Further flow cytometric analysis was performed on all cases to evaluate for doublets, using a plot of FSC area versus FSC height. The data acquisition was performed in a high-performance flow cytometer, FACSCanto II (BD Biosciences). The Infinicyt 1.7 software (Cytognos, Salamanca, Spain) was used for the analysis. According to the standardized protocol, human circulating EPCs were identified by a minimal antigenic profile that includes at least one marker of immaturity (CD34 and/or CD133), plus at least one marker of endothelial commitment (KDR).

Because EPCs are extremely rare events in peripheral blood, in order to increase the sensitivity of the method and the accuracy of our work we increased the total number of acquired events to at least 1 million per sample.

Four different populations of angiogenic cells were quantified: CD $34^{+}, \mathrm{CD}_{133^{+}}, \mathrm{CD}_{3} 4^{+} \mathrm{KDR}^{+}$and

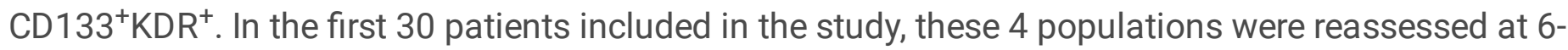
months of follow-up.

\section{Statistical analysis}

Statistical analyses were performed using SPSS software version 24 (IBM Corp., Armonk, NY, USA). Continuous variables were tested for normal distribution by Kolmogorov-Smirnov test and expressed as mean \pm standard deviation or median \pm interquartile range for parametric and nonparametric data, respectively. Categorical data are expressed as counts and percentages. For comparison of continuous data, we used unpaired Student t-test or nonparametric Mann-Whitney test for variables without a normal distribution. For the comparison of baseline and 6 months follow-up variables, the paired Student t-test or the Wilcoxon test was used, whichever appropriate. Categorical variables were compared with the chi-square test or with Fisher exact test as appropriate. Kaplan-Meier survival curves were used to evaluate the impact of EPCs levels on time-dependent clinical outcomes. Differences between pairs of survival curves were tested by the log-rank test. 
The relationship between variables was calculated using Pearson's or Spearman's correlation coefficient, whichever appropriate. A two-tailed $P$ value of $<0.05$ was considered statistically significant.

\section{Results}

\section{Baseline characteristics}

The baseline characteristics of the study population are presented in Table 1. Among the 50 patients with advanced HF, 11 patients (22\%) had an ischemic and 39 a non-ischemic etiology. Mean age was $61.7 \pm$ 10.5 years and the majority of patients were male $(64.0 \pm 48.5 \%)$. Seventy-seven percent of the patients were in NYHA class III, $10.6 \%$ in class II and $12.8 \%$ in ambulatory class IV before CRT. The global population had a LVEF of $23.3 \pm 6.8 \%$, a heart rate of $70.2 \pm 14.6$ beats/minute and a QRS duration of $143.4 \pm 29.0 \mathrm{~ms}$. 
Table 1

-Baseline characteristics in ischemic and non-ischemic patients.

\begin{tabular}{|c|c|c|c|}
\hline & $\begin{array}{l}\text { Ischemic etiology } \\
(n=11)\end{array}$ & $\begin{array}{l}\text { Non-ischemic etiology } \\
(n=39)\end{array}$ & $P$ value \\
\hline Age (years) ${ }^{a}$ & $61.5 \pm 9.4$ & $61.8 \pm 10.9$ & 0.920 \\
\hline Male gender $(\%)^{\mathrm{a}}$ & 100.0 & 53.8 & 0.004 \\
\hline Years since diagnosis $^{a}$ & $7.4 \pm 5.3$ & $5.8 \pm 6.0$ & 0.455 \\
\hline $\mathrm{NYHA}^{\mathrm{a}}$ & $2.9 \pm 0.3$ & $3.1 \pm 0.5$ & 0.390 \\
\hline $\mathrm{HR}$ (beats/min) ${ }^{\mathrm{a}}$ & $60.5 \pm 7.4$ & $72.8 \pm 15.0$ & 0.032 \\
\hline QRS (ms) ${ }^{\mathrm{a}}$ & $130.0 \pm 16.3$ & $147.7 \pm 31.1$ & 0.093 \\
\hline Diabetes (\%) & 36.4 & 18.4 & 0.209 \\
\hline CKD (\%) & 10.0 & 19.4 & 0.497 \\
\hline Hypertension (\%) & 55.6 & 26.5 & 0.098 \\
\hline Hyperlipidemia (\%) & 80.0 & 40.0 & 0.026 \\
\hline Statin (\%) & 90.9 & 50.0 & 0.016 \\
\hline Acetylsalicylic acid (\%) & 72.7 & 21.9 & 0.002 \\
\hline ACE-inhibitor (\%) & 72.7 & 71.9 & 0.958 \\
\hline AT-1 blocker (\%) & 9.1 & 15.6 & 0.600 \\
\hline Beta-blocker (\%) & 90.9 & 87.5 & 0.768 \\
\hline Spironolactone (\%) & 45.5 & 65.6 & 0.248 \\
\hline Furosemide (\%) & 90.9 & 100.0 & 0.088 \\
\hline Ivabradine (\%) & 9.1 & 15.6 & 0.600 \\
\hline Digoxin (\%) & 36.4 & 34.4 & 0.908 \\
\hline $\operatorname{LVESV}(\mathrm{mL})^{\mathrm{a}}$ & $157.7 \pm 35.0$ & $200.1 \pm 98.5$ & 0.193 \\
\hline $\operatorname{LVEDV}(\mathrm{mL})^{\mathrm{a}}$ & $218.3 \pm 37.9$ & $250.1 \pm 106.2$ & 0.363 \\
\hline $\operatorname{LVEF}(\%)^{a}$ & $26.5 \pm 6.3$ & $22.3 \pm 6.8$ & 0.078 \\
\hline $\operatorname{BNP}(p g / m L)^{a}$ & $381.1 \pm 330.5$ & $550.0 \pm 602.5$ & 0.458 \\
\hline CRT-D versus CRT-P (\%) & $100.0 / 0.0$ & $81.3 / 18.8$ & 0.308 \\
\hline
\end{tabular}


aMean \pm standard deviation.

ACE = angiotensin-converting enzyme; $C K D$ = chronic kidney disease; BNP = brain natriuretic peptide; CRT-D = cardiac resynchronization therapy-defibrillator; CRT-P = cardiac resynchronization therapypacemaker; HR = heart rate; LVEDV = left ventricular end-diastolic volume; LVEF = left ventricular ejection fraction; LVESV = left ventricular end-systolic volume; NYHA = New York Heart Association.

Regarding the type of device implanted, the proportion of CRT-D and CRT-P was respectively 85.7 and $14.3 \%$. Regarding the chronic medication, $72.1 \%$ of the patients were under angiotensin converting enzyme inhibitors (ACE inhibitors), 88.4\% under beta adrenergic blockers (BB), 60.5\% under spironolactone, $97.7 \%$ under furosemide, $34.9 \%$ under digoxin, $60.5 \%$ under statins, $34.9 \%$ under aspirin (ASA), and $14.0 \%$ under ivabradine. As expected, the proportion of patients treated with statins and ASA was significantly higher in the group of patients with ischemic cardiomyopathy (ICM).

Patients with ICM were more frequently male and had a higher proportion of cardiovascular risk factors (diabetes, hypertension and hyperlipidemia) than patients with non-ischemic cardiomyopathy (DCM) (Table 1). Moreover, the heart rate was significantly lower in ICM compared to DCM.

Patients with DCM tended to have a lower LVEF value when compared to patients with ICM $(22.3 \pm 6.8 \%$ versus $26.5 \pm 6.3 \%, p=0.078$, respectively) (Table 1 ).

\section{Circulating EPCs levels according to ischemic and non- ischemic etiology}

There were no statistically significant differences in levels of circulating $\mathrm{CD} 34^{+}, \mathrm{CD} 133^{+}$or $\mathrm{CD} 133^{+} \mathrm{KDR}^{+}$ cells between ischemic and non-ischemic patients (Fig. 1). However, the CD133 ${ }^{+}$angiogenic cells tended to circulate in lower numbers in patients with ICM compared to patients with an DCM (Fig. 1A). Levels of circulating $\mathrm{CD} 34^{+} \mathrm{KDR}^{+}$EPCs were significantly lower in patients with ICM (Fig. 1B).

\section{Long-term outcome after CRT}

At 6-month follow-up, we observed a significant improvement in LVEF (from $23.7 \pm 6.8 \%$ to $31.5 \pm 11.0 \%$, $\mathrm{p}<0.001$ ), with a significant decrease in left ventricular volumes (from $189.0 \pm 89.7 \mathrm{ml}$ to $156.4 \pm 96.5 \mathrm{ml}$ in LVESV, $p=0.004$ ). However, $42 \%$ of patients did not respond favourably to CRT according to remodelling criteria.

The proportion of non-responders to CRT tended to be higher in patients with an ischemic etiology by comparison with non-ischemic patients ( $64 \%$ versus $35 \%, p=0.098)$ (Table 2$)$. 
Table 2

- Comparison of clinical evolution between ischemic and non-ischemic patients

\begin{tabular}{|llll|}
\hline & $\begin{array}{l}\text { Ischemic etiology } \\
(\mathbf{n}=\mathbf{1 1})\end{array}$ & $\begin{array}{l}\text { Non-ischemic etiology } \\
(\mathbf{n}=\mathbf{3 9})\end{array}$ & P value \\
\hline Number of hospitalizations & $1.8 \pm 2.0$ & $0.8 \pm 1.3$ & 0.052 \\
\hline Rehospitalization for HF (\%) & 63.6 & 38.5 & 0.137 \\
\hline Time Until First Release (months) & $46.8 \pm 40.1$ & $53.1 \pm 35.4$ & 0.429 \\
\hline CV death (\%) & 36.4 & 35.9 & 0.977 \\
\hline Heart transplantation (\%) & 9.1 & 2.6 & 0.329 \\
\hline Responders (\%) & 36.4 & 64.7 & 0.098 \\
\hline
\end{tabular}

Regarding long-term clinical outcome (mean follow-up of $5.4 \pm 2.3$ years), 18 patients died: $5 / 29$ (17\%) in the responder group and $13 / 21(61 \%)$ in the non-responder group $(p=0.019)$. Two patients underwent heart transplantation (one responder and one non-responder) and 22 patients were re-hospitalized due to HF: $8 / 29(28 \%)$ in responder group and $14 / 21(67 \%)$ in non-responders to CRT ( $p=0.039)$.

During follow-up, there were no statistically significant differences in mortality rate, or heart transplantation rate between ischemic and non-ischemic patients (supplementary data). However, patients with ICM tended to be more often hospitalized due to HF than DCM patients (mean number of hospitalizations: $1.8 \pm 2.0$ vs $0.8 \pm 1.3, p=0.052$, respectively and hospitalization rate: $63.6 \%$ vs $38.5 \%, p$ $=0.137$, respectively (Table 2 ).

There were no significant differences in baseline EPCs levels among patients who were alive and patients who died during long-term follow-up nor between patients who were rehospitalized for heart failure management or not (supplementary data). Additionally, there was no correlation between baseline EPCs levels and time to rehospitalization, number of rehosts, or time to death and survival curves for mortality and rehospitalization due to HF were not significantly different between patients with EPCs numbers under or above the media (supplementary data).

\section{Evolution of EPC levels after CRT}

Six-months after CRT patients presented significantly higher levels of both $C D 34^{+} \mathrm{KDR}^{+}$and $\mathrm{CD} 133^{+} \mathrm{KDR}^{+}$ EPCs than before the implantation (Fig. 2). However, we did not find significant differences in the degree of increase in EPCs between responders and non-responders to CRT.

\section{Discussion}

To the best of our knowledge, this is the first study assessing the impact of CRT on circulating EPCs levels. 
The main findings of the present work can be summarized as follows. First, the etiology of heart failure seems to influence EPCs levels, with lower number of circulating EPCs in the ischemic patients. Second, circulating levels of $\mathrm{CD} 34^{+} \mathrm{KDR}^{+}$and $\mathrm{CD} 133^{+} \mathrm{KDR}^{+}$cells significantly increase after $\mathrm{CRT}$, independently of patient's response. Third, baseline EPCs numbers seem not to correlate with long-term outcome after CRT.

Heart failure with reduced ejection fraction (HFrEF) is a very common disease with a poor prognosis. The prevalence of HF can be estimated at approximately $1-2 \%$ of the adult population in developed countries and the incidence approaches $5-10$ per 1000 persons per year. ${ }^{10}$

Over the last two decades, CRT has revolutionized the treatment of selected patients who have HFrEF. CRT improves cardiac performance in appropriately selected patients and reduces morbidity and mortality. ${ }^{11}$ Several studies have demonstrated the efficacy of CRT in counteracting ventricular remodeling through the recovery of synchronous muscle contractility. ${ }^{12}$ However, the exact mechanisms leading to the long-term benefits of CRT are not yet fully understood and other mechanisms beyond left ventricular reverse remodeling are likely involved, explaining the discordance frequently observed between clinical and remodeling response to CRT and also between CRT response and long-term outcomes. ${ }^{13}$

End-stage HF is the final common pathway for several different diseases, with ischemic aetiology being responsible for the vast majority of cases, in developed countries. ${ }^{14}$

Previous studies have suggested that patients with ischemic etiology have a lower probability of response to CRT than non-ischemic patients. ${ }^{8,15}$ The reasons for the lack of response to CRT are not well understood. In ischemic etiology, LV desynchrony may be related to segmental wall motion abnormalities due to the presence of myocardial scars or perfusion defects that cannot be resynchronized. ${ }^{15}$ Here, we verify that patients with ICM express significantly lower levels of circulating EPCs, suggesting that this pauperization may justify why ICM patients typically benefit less from CRT. However, several studies conducted in recent years have found that the benefits of CRT appear to be similar in HF regardless of the underlying cause. Therefore, presently, the decision to indicate CRT is not influenced by the etiology of $\mathrm{HF}^{14}$

Endothelial dysfunction has been extensively reported in patients with HF. ${ }^{16}$ Endothelial damage or ischemia leads to liberation of several mediators, such as VEGF, stromal cell-derived factor 1 (SDF-1) or nitric oxide synthase (NOS). This cascade activation seems to stimulate the proliferation of EPCs in bone marrow and their release to bloodstream. Circulating EPCs adhere to the injured endothelium, playing a crucial role in vascular repair. ${ }^{17}$ During recent years, accumulating evidence revealed that circulating EPCs showed reduced numbers and functional impairment within several cardiovascular diseases. Valgimigli et al. were the first to evaluate the role of circulating EPCs in HF patients. They showed decreasing EPC levels with more advanced stages of congestive HF indicated by higher NYHA classes and elevated NT-proBNP levels. ${ }^{18}$ Nonaka-Sarukawa et al. also showed that HF patients present lower EPCs counts than controls. ${ }^{2}$ The reduction of circulating EPCs levels in advanced HF can be justified by 
diffuse and severe endothelial damage. However, conflicting results about the behaviour of circulating EPCs in advanced HF have been published. Theiss et al. found that circulating EPCs were lower in patients with ICM than DCM but still higher than healthy controls. ${ }^{19}$ Heeschen et al. observed a functional impairment of bone marrow-derived EPCs leading to a reduced migratory capacity into the circulation of patients with ischemic HF compared to healthy controls. ${ }^{20}$ However, findings from other investigators groups indicate that the etiology of HF does not differentially affect circulating EPCs. ${ }^{18}$ In our study, despite the greater use of statins (a stimulus for EPCs) in patients with ischemic etiology, they showed significantly lower levels of circulating EPCs when compared to non-ischemic patients. This reduced circulating EPCs levels were observed for both the $C D 34^{+} \mathrm{KDR}^{+}$cells and for the more immature $\mathrm{CD} 133^{+} \mathrm{KDR}^{+}$population. That difference could potentially explain why ICM patients typically benefit less from CRT and the worse prognosis generally associated with ischemic etiology compared to nonischemic causes of HF.

\section{Long-term outcome after CRT}

Low circulating EPC levels are associated with adverse outcomes in patients with coronary artery disease. ${ }^{5}$ However, regarding CHF, Michowitz et al. showed that higher levels of EPCs independently predicted all-cause mortality. ${ }^{21}$ In contrast, Koller et al. showed that EPCs defined as CD $34^{+}$CD $45^{\text {dim }} \mathrm{KDR}^{+}$ cells were a strong and independent inverse predictor of mortality in patients with chronic $\mathrm{HF}^{22}$ Similarly, Samman Tahhan et al. demonstrated that lower EPCs counts were strongly and independently predictive of mortality. ${ }^{23}$ On other hand, another study found that $C D 34^{+} \mathrm{KDR}^{+}$levels were not related with the risk of mortality, composite outcomes, or hospital admissions in patients with ambulatory left ventricular ejection fraction $<40 \% .{ }^{24}$ However, the potential impact of circulating EPCs on clinical outcomes after CRT had not yet been studied. In our study, baseline EPCs levels were not related with long-term outcomes in HF submitted to CRT.

\section{Evolution of EPC levels after CRT}

An important observation of our study is that numbers of both EPCs populations (CD34 ${ }^{+} \mathrm{KDR}^{+}$cells and $\mathrm{CD} 133^{+} \mathrm{KDR}^{+}$cells) significantly increase after CRT. We can speculate that this increase in EPCs is a result of effective CRT which may translate in an improved capacity of endothelial repair mediated by EPCs. However, the significance of this finding remains to be determined.

In recent years the role of EPCs in cardiovascular disease as well as the interplay between inflammation and endothelial progenitor cell biology have been discussed. In patients at an increased cardiovascular risk (diabetes mellitus, systemic hypertension and hyperlipidemia) EPCs show a decreased proliferative capacity, and present reduced levels in peripheral circulation. ${ }^{6,25}$ In patients with advanced CHF, the majority of studies indicate that circulating EPCs levels are profoundly decreased. ${ }^{2,18,20}$

$\mathrm{HF}$ is characterized by a chronic inflammatory status with elevated pro-inflammatory cytokines, such as tumor necrosis factor (TNF)- $a$, interleukin (IL)-1 $\beta$ and IL-6. This inflammatory milieu can negatively 
impact on circulating EPCs. ${ }^{26,27}$

Previous studies have shown that CRT reduces the inflammatory milieu of chronic HF. ${ }^{26,27}$ Theodorakis et al. showed that IL-6 and TNF-a were reduced after 3 months of biventricular pacing. ${ }^{26}$ In the present study, circulating EPCs significantly increase after CRT. Therefore, we can speculate that this antiinflammatory action of CRT can be translated into increase in circulating levels of $\mathrm{CD}^{+} 4^{+} \mathrm{KDR}^{+}$and CD $133^{+}$KDR EPCs. However, these findings need confirmation and possible mechanisms to explain this association need further investigation.

\section{Limitations}

This study had a relatively small sample size and future larger studies would be important to confirm that circulating EPCs do not influence long-term prognosis of HF patients submitted to CRT. Another limitation of the study is the absence of a healthy control group, which could help to understand whether or not the increase in EPCs levels after CRT means a return towards normal levels.

We were not able to explore other functional characteristics of EPCs that might provide further understanding about the role of CRT on EPCs response and its contribution to HF pathogenesis.

\section{Conclusions}

Our study shows that patients with ICM present a pauperization in the EPCs pool and it suggests that CRT may increase circulating EPCs levels. Additionally, reduced baseline EPCs numbers seem not to influence long-term outcomes after CRT. However, further studies are warranted to better understand the role of EPCs in advanced HF and its potential relation to the beneficial effects of CRT.

\section{Abbreviations}

ACE-inhibitors - Angiotensin Converting Enzyme Inhibitors

ASA- Aspirin

CRT - Cardiac Resynchronization Therapy

DCM - Dilated Cardiomyopathy (non-ischemic cardiomyopathy)

EDTA - Ethylenediaminetetraacetic acid

EPCs - Endothelial Progenitor Cells

FITC - Fluorescein isothiocyanate

HF - Heart Failure 
HFrEF - Heart failure with reduced ejection fraction

ICM - Ischemic Cardiomyopathy

IL - Interleukin

LVEDV - Left Ventricular End-Diastolic Volume

LVEF - Left Ventricular Ejection Fraction

LVESV - Left Ventricular End-Systolic Volume

NYHA - New York Heart Association

NOS - Nitric Oxide Synthase

PE - Phycoerythrin

SDF-1 - Stromal Cell-Derived Factor 1

TNF - Tumor Necrosis Factor

VEGF-R2 - type 2 Vascular Endothelial Growth Factor Receptor

\section{Declarations}

Ethics approval and consent to participate: All study patients signed an informed consent, the study was approved by the ethics committee of Centro Hospitalar e Universitário de Coimbra and is in accordance with the criteria of the Declaration of Helsinki

Consent for publication: Not applicable.

Availability of data and materials: The datasets used and/or analysed during the current study are available from the corresponding author on reasonable request.

Competing interests. The authors declare that they have no competing interests.

Funding: This work was supported by an unrestricted research grant from St Jude Medical and consulting fees from Boston Scientific.

Authors' contributions. GC, analysed and quantified EPCs numbers; JM, performed the statistical analysis and was the main contributor in writing the manuscript; Pedro Sousa, collected the clinical data of patients included in the study and critically analysed the results; MV, was responsible for the collection of the data regarding clinical follow-up and critically analysed the results; JC, discussed the results and gave an important contribution in the revision of the manuscript; $L E$, analysed the clinical data and gave an important contribution in the revision of the manuscript; AP, analysed and quantified EPCs numbers; LG, 
contributed to the collection clinical data and design of the work; CFR, critically discussed all data and gave an important contribution in the revision of the manuscript; NA, designed the project and was responsible for the supervision of all the work. All authors read and approved the final manuscript

\section{Acknowledgements section: Not applicable.}

\section{References}

1. Fischer D, Rossa S, Landmesser U, Spiekermann S, Engberding N, Hornig B, et al. Endothelial dysfunction in patients with chronic heart failure is independently associated with increased incidence of hospitalization, cardiac transplantation, or death. Eur Heart J. 2005;26(1):65-9.

2. Nonaka-Sarukawa $M$, Yamamoto $K$, Aoki H, Nishimura $Y$, Tomizawa $H$, Ichida $M$, et al. Circulating endothelial progenitor cells in congestive heart failure. Int J Cardiol. 2007;119(3):344-8.

3. Hristov M, Erl W, Weber PC. Endothelial progenitor cells: Mobilization, differentiation, and homing. Arterioscler Thromb Vasc Biol. 2003;23(7):1185-9.

4. Chu K, Jung KH, Lee ST, Park HK, Sinn DI, Kim JM, et al. Circulating endothelial progenitor cells as a new marker of endothelial dysfunction or repair in acute stroke. Stroke. 2008;39(5):1441-7.

5. Schmidt-Lucke C, Rössig L, Fichtlscherer S, Vasa M, Britten M, Kämper U, et al. Reduced number of circulating endothelial progenitor cells predicts future cardiovascular events: Proof of concept for the clinical importance of endogenous vascular repair. Circulation. 2005;111(22):2981-7.

6. Werner N, Kosiol S, Schiegl T, Ahlers P, Walenta K, Link A, et al. Circulating Endothelial Progenitor Cells and Cardiovascular Outcomes. N Engl J Med. 2005;353(10):999-1007.

7. Abraham W, Fisher W, Smith A, Delurgio D, Leon A, Loh E, et al. Cardiac Resynchronization in Chronic Heart Failure. N Engl J Med. 2002;346(24):1845-53.

8. Díaz-Infante E, Mont L, Leal J, García-Bolao I, Fernández-Lozano I, Hernández-Madrid A, et al. Predictors of lack of response to resynchronization therapy. Am J Cardiol. 2005;95(12):1436-40.

9. Antonio N, Soares A, Carvalheiro T, Fernandes R, Paiva A, Ventura M, et al. Circulating endothelial progenitor cells as a predictor of response to cardiac resynchronization therapy: The missing piece of the puzzle? PACE - Pacing Clin Electrophysiol. 2014;37(6):731-9.

10. Bleumink GS, Knetsch AM, Sturkenboom MCJM, Straus SMJM, Hofman A, Deckers JW, et al. Quantifying the heart failure epidemic: Prevalence, incidence rate, lifetime risk and prognosis of heart failure - The Rotterdam Study. Eur Heart J. 2004;25(18):1614-9.

11. Cleland JG, Abraham WT, Linde C, Gold MR, Young JB, Claude Daubert J, et al. An individual patient meta-analysis of five randomized trials assessing the effects of cardiac resynchronization therapy on morbidity and mortality in patients with symptomatic heart failure. Eur Heart J. 2013;34(46):3547-56.

12. Cleland JGF, Calvert MJ, Verboven Y, Freemantle N. Effects of cardiac resynchronization therapy on long-term quality of life: An analysis from the CArdiac Resynchronisation-Heart Failure (CARE-HF) study. Am Heart J. 2009;157(3):457-66. 
13. Boidol J, Średniawa B, Kowalski O, Szulik M, Mazurek M, Sokal A, et al. Many response criteria are poor predictors of outcomes after cardiac resynchronization therapy: Validation using data from the randomized trial. Europace. 2013;15(6):835-44.

14. Ponikowski P, Voors AA, Anker SD, Bueno H, Cleland JG, Coats AJ, et al. 2016 ESC Guidelines for the diagnosis and treatment of acute and chronic heart failure. Eur Heart J. 2016;37:2129-200.

15. Chen Y, Duan C, Liu F, Shen S, Chen P, Bin J. Impact of etiology on the outcomes in heart failure patients treated with cardiac resynchronization therapy: A meta-analysis. PLoS One. 2014;9(4):1-10.

16. Kubo SH, Rector TS, Bank AJ, Williams RE, Heifetz SM. Endothelium-dependent vasodilation is attenuated in patients with heart failure. Circulation. 1991;84(4):1589-96.

17. Hristov $M$, Weber $C$. Endothelial progenitor cells: Characterization, pathophysiology, and possible clinical relevance. J Cell Mol Med. 2004;8(4):498-508.

18. Valgimigli M, Rigolin GM, Fucili A, Della Porta M, Soukhomovskaia O, Malagutti P, et al. CD34+ and endothelial progenitor cells in patients with various degrees of congestive heart failure. Circulation. 2004;110(10):1209-12.

19. Theiss HD, David R, Engelmann MG, Barth A, Schotten K, Naebauer M, et al. Circulation of CD34+ progenitor cell populations in patients with idiopathic dilated and ischaemic cardiomyopathy (DCM and ICM). Eur Heart J. 2007;28(10):1258-64.

20. Heeschen C, Lehmann R, Honold J, Assmus B, Aicher A, Walter DH, et al. Profoundly Reduced Neovascularization Capacity of Bone Marrow Mononuclear Cells Derived from Patients with Chronic Ischemic Heart Disease. Circulation. 2004;109(13):1615-22.

21. Michowitz Y, Goldstein E, Wexler D, Sheps D, Keren G, George J. Circulating endothelial progenitor cells and clinical outcome in patients with congestive heart failure. Heart. 2007;93(9):1046-50.

22. Koller L, Hohensinner P, Sulzgruber P, Blum S, Maurer G, Wojta J, et al. Prognostic relevance of circulating endothelial progenitor cells in patients with chronic heart failure. Thromb Haemost. 2016;116(2):309-16.

23. Samman Tahhan A, Hammadah M, Sandesara P, Hayek S, Kalogeropoulos A, Alkhoder A, et al. Progenitor Cells and Clinical Outcomes in Patients with Heart Failure. Circ Heart Fail. 2017;10(8):e004106.

24. Alba AC, Lalonde SD, Rao V, Walter SD, Guyatt GH, Ross HJ. Changes in circulating progenitor cells are associated with outcome in heart failure patients: A longitudinal study. Can $\mathrm{J}$ Cardiol. 2013;29(12):1657-64.

25. Vasa M, Fichtlscherer S, Aicher A, Adler K, Urbich C, Martin H, Zeiher AM DS. Number and migratory activity of circulating endothelial progenitor cells inversely correlate with risk factors for coronary artery disease. Circ Res. 2001;6(89 (1)):E1-7.

26. Theodorakis GN, Flevari P, Kroupis C, Adamopoulos S, Livanis EG, Kostopoulou A, et al. Antiinflammatory effects of cardiac resynchronization therapy in patients with chronic heart failure. Pacing Clin Electrophysiol. 2006;29(3):255-61. 
27. Stanciu AE, Vatasescu RG, Stanciu MM, lorgulescu C, Vasile Al, Dorobantu M. Cardiac resynchronization therapy in patients with chronic heart failure is associated with anti-inflammatory and anti-remodeling effects. Clin Biochem. 2013;46(3):230-4.

\section{Figures}

A

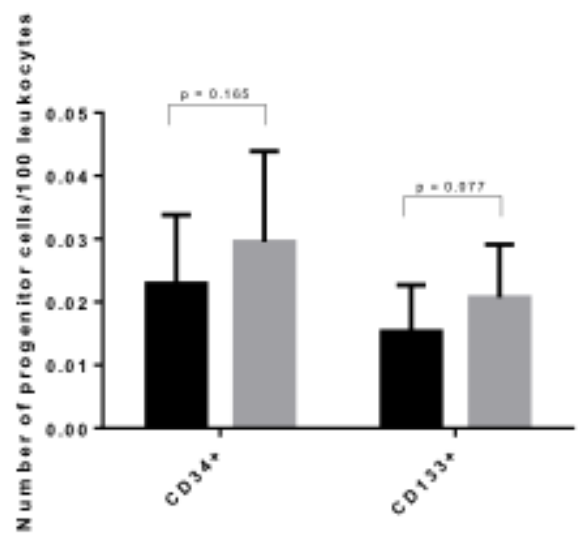

B

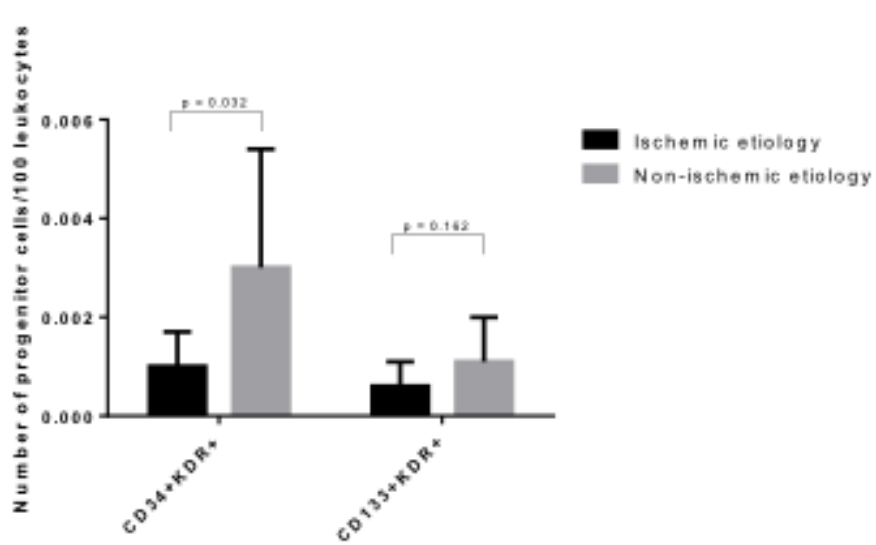

\section{Figure 1}

EPCs levels according to ischemic or non-ischemic etiology. (A) Comparison of circulating levels of angiogenic CD34+ and CD133+ cells between ischemic and non-ischemic patients. (B) Comparison of CD34+KDR+ and CD133+KDR+ EPCs levels between ischemic and non-ischemic patients. EPCs, endothelial progenitor cells 


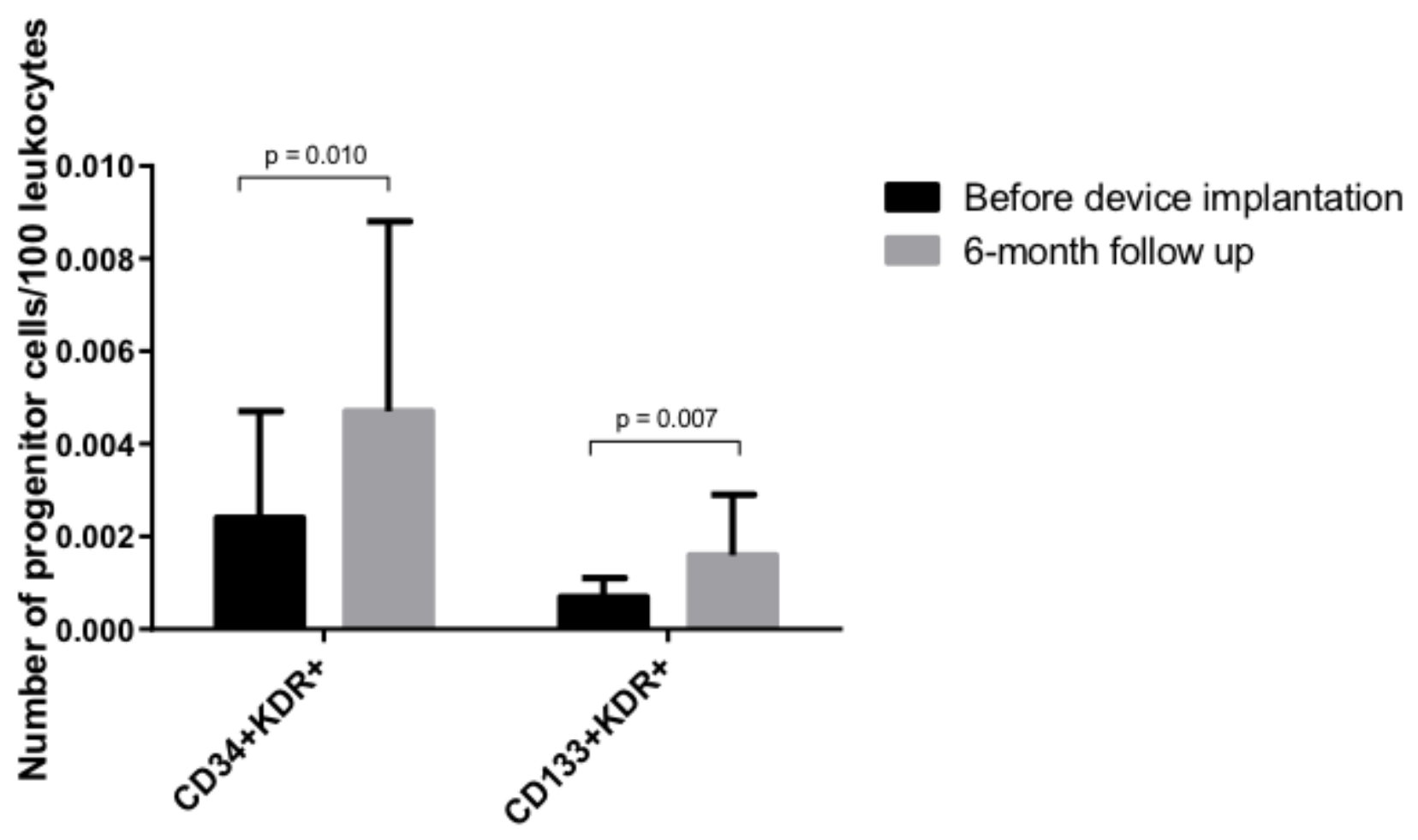

Figure 2

Evolution of EPCs levels from baseline to six months after implantation

\section{Supplementary Files}

This is a list of supplementary files associated with this preprint. Click to download.

- SupplementaryFigure.tiff 